\title{
A Survey paper on Object Cutting problem using Dynamic programming and Artificial Intelligence
}

\author{
Manthan Shah \\ B.Tech (IT) Student \\ Department of \\ Information \& Technology \\ Charotar University of Science \& \\ Technology \\ (CHARUSAT)
}

\author{
Shivani Bhakta \\ B.Tech(IT) Student \\ Department of Information \& \\ Technology \\ Charotar University of Science \& \\ Technology \\ (CHARUSAT)
}

\author{
Sonal Rami \\ Assistant Professor \\ Department of Information \& \\ Technology \\ Charotar University of Science \& \\ Technology \\ (CHARUSAT)
}

\begin{abstract}
This paper focuses on the intelligent object cutting approach for optimizing manufacturing problem in manufacturing industries. The main motive behind inscribing this paper is to scale back or minimize the wastage from the unessential cutting of stuff because it has been a key issue. In other words, minimizing the wastage and maximizing the profit is the main goal. As per the current scenario in the manufacturing industries, the manufacturers are observing a huge loss of materials resulting in minimized profit. So, in order to overcome this problem an algorithm must be designed which gives an optimal solution for object cutting problem.
\end{abstract}

\section{Keywords}

Manufacturing problem, Heuristic function, Optimization

\section{INTRODUCTION}

The first question arises in every mind is that is what is AI? Human Intelligence Exhibited by Machines. . So, the solution to the current question is that the theory and development of pc systems ready to perform tasks unremarkably requiring human intelligence, like seeing, speech recognition, decision-making, and translation between languages. In other words, the field of AI analysis defines itself because the study of "intelligent agents": any device that perceives its atmosphere and takes actions that maximize its likelihood of success at some goal. AI analysis is split into subfields that specialize in specific issues or on specific approaches or on the utilization of explicit specific selected tool or towards satisfying particular applications. The other question can be raised of optimality. What is optimality? The answer to this is it is a linguistic model proposing that the discovered styles of language arise from the interaction between conflicting constraints.

\section{LITERATURE REVIEW}

Paper: Optimal Allocation of Two-Dimensional Irregular Shapes Using Heuristic Search Methods:

Heuristic search method and ALGOLW algorithm is used.

Assumptions which are made in the paper are:

- The items are irregular polygons while not holes and therefore the sheet may be a parallelogram, massive enough to contain all the demanded items regardless the standard of the answer.
- The items are often rotated; should they need to lie entirely at intervals the sheet and must not overlap.

The first step consists in associating to the matter a collection of states and operators remodeling one state into another.

Two categories of algorithms are often distinguished in line with whether or not or not they use data regarding the conferred downside to hurry up the search of the best path. This data is termed heuristic information: once it's used, we tend to name a heuristic search methodology. Hart, Nilsson, associate degreed Raphael introduced an estimate that a node $\mathrm{n}$ are often on the optimum answer path, known

as analysis perform, outlined as $\mathrm{f}(\mathrm{n})=\hat{\mathrm{g}}(\mathrm{n})+\mathrm{h}(\mathrm{n})$. Here $\hat{\mathrm{g}}(\mathrm{n})$ is associate degree estimate of $g(n)$, the shortest path price from the beginning node to $n$, associate degreed $\hat{h}(n)$ an estimate of $h(n)$, the shortest path price from $n$ to the ultimate node. At every iteration, the node $\mathrm{n}$ whose total estimate $f(n)$ is smallest are going to be elite. Representation of allocation problem: Let an immediate allocation $\mathrm{Ai}$ on the resource be associate ordered strive of lists $(\mathrm{P}, \mathrm{Q})$, such $\mathrm{P}$ contains the position and orientation of all the things assigned, and letter contains the unallocated things. At the beginning $\mathrm{P}$ is empty and Ao is called the initial allocation; whereas, once letter is empty, $\mathrm{Ai}$ is called a final allocation and will be the solution of the matter.

An allocation Ai+1 may be a successor of $\mathrm{Ai}$ if it's obtained transcription one altogether the unallocated things in Ai according to a prefixed placement policy. The position policy ought to satisfy all the specified constraints on the layout, e.g., a current piece need to be organized inside the resource so on not overlap the preceding ones. ${ }^{[1]}$

\section{Techniques to Increase Heuristic Search Power:}

\section{Evaluation Function:}

In order to preserve the "admissibility" of the search algorithms, i.e., the property of finding a minimal-cost path, the evaluation function used to choose from the list of unplaced pieces the one to place next, should be expressed as sum of $\mathrm{g}$ and $\mathrm{h}$, two cost estimate functions.

\section{Successor Limitation:}

- For every however unsuccessful piece the location policy is applied to any or all doable orientations, however solely the 
one that produces

the left lowest allocation are thought of in step two. Ties square measure resolved in favor of the allocation with the minimum extension to the proper. - From the items chosen within the previous step, solely a set range (parameter MAXGENERATED) of the left allotted items square measure preserved for step three. Ties square measure resolved in favor of the piece with the utmost extension to the proper. - For the allocation obtained within the previous step, a set range of successors (parameter NSUCCESSORS) are generated per the worth of $f$. Ties square measure resolved in favor of the piece bestowed initial.

- Since the list of generated nodes is finite (parameter LIST SIZE), once it becomes full, the tree to this point generated by the search is cropped by erasing the node with the best $\mathrm{f}$ worth.

\section{Evaluation Function Discretization:}

During the execution of the program the evaluating perform assumes massive values distributed during a slim interval. So it will happen that a successor of the present enlarged node is by a number of units worse than all the others within the list generated. During this case, it's preferred to continue the search on identical path as so much because the worth of the analysis perform continues to be slightly higher. This has been accomplished by introducing a parameter exactitude that represents the amount of the foremost important digits of the $\mathrm{f}$ values to be thought-about by the algorithmic rule.4. Expansion Band: When the search is at $\mathrm{k}^{\text {th }}$ level, the next node to be expanded has to be at a level not lower than $(k-t)$, where $\mathrm{t}$ is a given threshold.

\section{Termination condition:}

The search doesn't terminate once all the items are allotted, however once the list of generated nodes is empty.

\section{Profile Simplification:}

At every step the profile is simplified so as to exclude all the areas on the left aspect of the vertical line through the left purpose of the last allotted items. The program uses some operators for the things and profile descriptions:

Piece [Piece-Description]: Inputs the figure description of a piece. a piece is delineate by providing a list of the points (vertices) of the figure approximation given throughout a counterclockwise $(\mathrm{CCW})$ direction.

No-Fit-Polygon [Piece A, Piece B]: Computes the no fitpolygon (NFP) for the given mix of things with the given orientations. The NFP completely describes all those positions where the indicator of $\mathrm{B}$ may even be placed thus on possess B touching a short while not overlapping it.

Allocation Region [Resource, Piece]: Computes the perimeter of the area in which the reference point of the piece can validly fall.

Envelope [Profile]: Closes the profile with the border of the resource.
Allocation purpose [Profile, Piece]: Computes the left lower allocation purpose of the piece with reference to the present profile.

New Profile [Profile, Piece, and Allocation Point]: Computes the new profile ensuing by a mix of the recent one and also the piece placed with the purpose within the allocation point.

Normalization [Piece]: Normalizes the outline of the items in such how that the indicator, the left lower vertex and also the origin of the coordinates can coincide.

Area [Piece]: Computes the area of the piece.

Flip [Piece]: Reflects the shape about the vertical axis through the reference point.

Rotate [Piece, Theta]: Rotates the piece through of "theta" degrees $\mathrm{CCW}$ about the reference point.

These facilities are enforced to manage the program execution throughout its development; they might be improved by the utilization of Associate in nursing interactive show system wherever Associate in nursing operator will interactively modify the layout.

In the initial one, the search payoff during a consecutive and settled manner, i.e., once a chunk is placed in some position, it'll not be removed any longer. The opposite manner of manufacturing the answer, permits the method to double back, i.e., to suspend this path search and to develop a brand new one.

Paper: Hyper-heuristics and Classifier Systems for solving 2D-Regular Cutting Stock Problems Set of heuristic functions and artificial intelligence are used.

By using different sets of heuristics as next fit, first fit, best fit, worst fit, almost worst fit, first fit decreasing, next fit decreasing and Djang fitch. ${ }^{[2]}$

The representations are done step-wise as:

- Height representation

- Width representation

- Area representation

- Ratio of items to be cut

Then the heuristics is followed by Selection Heuristics $(\mathrm{SCH})$ and Placement Heuristics (PMH).

Each part has some proportion of items which is divided to the respective bits.

Then by matching set and prediction array, action set is formed which later on takes the best classifier to perform the action of cutting. ${ }^{[5]}$

After this, the rewards are applied according to single-step and multi-step in which if process is completed in single-step then reward is paid after every combination of heuristics and in multi-step reward is paid after delivering a complete solution.

Paper: Optimizing Allocation of Two Dimensional Irregular Shapes using an Agent Based Approach

$\mathrm{VC}++6.0$ programming language is used. 
A relation can only be made when both vertices and edges of two shapes lay on each other.

From this, a relation between 2 shapes are often established by their common vertices and customary edges. ${ }^{[3]}$

After a relation is formed, there are no overlapping parts and without this condition a relation would not be possible.

To avoid construction of duplicate copies following two restrictions are required:

(1) Agent A and B should not have an acquaintance of an equivalent form.

(2) Once A and B kind their relation, none of them or their friends might overlap.

Once a group is duplicated, the original and its copies explode and during explosion new relations trash and therefore the relations that have lasted longer are additional probable for the later teams.

The relations with average results will be killed and removed from the population to increase the variety.

During implementation, it's ascertained that the wasted space is a smaller amount than a pair of $0.5 \%$ and every one results ar obtained in but quarter-hour of the method. ${ }^{[6]}$

\section{Motivation from literature review}

- As per the current scenario in the manufacturing industries, the client as well as the manufacturer are observing a huge loss of raw materials resulting in minimized profit.

- As the process includes a lot of man power as well as machineries which may increase the chances of occurrence of errors due to which optimal solution is not obtained.

- Reducing wastage from the needless cutting of staple becomes a key issue.

- To develop an algorithm which provides the solution for above stated problem in order to obtain the optimal solution.

\section{Paper: Improved Cut Sequences for Partitioning Based Placement}

In this paper, two partitioning tools i.e. Capo and Feng Shui are compared to obtain cut sequence for the object cutting problem.

Both tools complete the placement process by performing branch-and-bound reordering of cells within a row.

The main focus of this paper is that one factor which differentiates the cut sequence of both the tools due to which the result differs. ${ }^{[4]}$

For optimal generation of cut sequences, estimation of cut sizes and wire lengths is a must requirement.

So, Rent's rule is used which gives propagated terminals by a formula $\mathrm{T}=\mathrm{k} * \mathrm{C}^{\mathrm{p}}$

From this rule, number of nets split between two sub regions can be determined so that according to this, co-ordinates can be obtained.

Other approach is Dynamic Programming Formulation in which each partitioning splits a region into several new ones, with the total wire length depending on the contribution of nets that are cut by the partition, and also on the wire lengths introduced by the sub regions.

To indicate the optimal wire length for an $\mathrm{r}$ by $\mathrm{c}$ region, a formula is used which is

$$
W L(r, c)=\min \left\{\begin{array}{l}
\text { Horizontal cuts } \\
C L(1, c, r-1, c)+W L(1, c)+W L(r-1, c) \\
C L(2, c, r-2, c)+W L(2, c)+W L(r-2, c) \\
\ldots \\
C L(r-1, c, 1, c)+W L(r-1, c)+W L(1, c) \\
\text { Vertical cuts } \\
C L(r, 1, r, c-1)+W L(r, 1)+W L(r, c-1) \\
C L(r, 2, r, c-2)+W L(r ; 2)+W L(r, c-2) \\
\ldots \\
C L(r, c-1, r, 1)+W L(r, c-1)+W L(r, 1)
\end{array}\right.
$$

Then by applying this method, an optimal cut sequence table is generated which has two properties:

- In all cases, optimal sequences include bisections, and not partitions of arbitrary balance.

- If the ratio of rows to columns exceeds a threshold value, and optimal sequence partitions horizontally; otherwise, an optimal sequence partitions vertically.

\section{Comparative Study:}

\begin{tabular}{|c|c|c|}
\hline+ & Title & Learning Outcome \\
\hline 1 & $\begin{array}{l}\text { Optimal } \\
\text { Allocation } \\
\text { of Two- } \\
\text { Dimensiona } \\
1 \quad \text { Irregular } \\
\text { Shapes } \\
\text { Using } \\
\text { Heuristic } \\
\text { Search } \\
\text { Methods. }\end{array}$ & $\begin{array}{l}\text { An optimistic approach has been } \\
\text { described in order to allocate/cut irregular } \\
\text { shaped two-dimensional object. Using } \\
\text { heuristic } \\
\text { search technique, associate algorithmic } \\
\text { rule has been introduced so as to offer the } \\
\text { probable answer in terms of quality i.e. } \\
\text { less } \\
\text { of material and economical in computing } \\
\text { time. }\end{array}$ \\
\hline 2 & $\begin{array}{l}\text { Hyper- } \\
\text { Heuristics } \\
\text { and } \\
\text { Classifier } \\
\text { for solving } \\
\text { 2-D Regular } \\
\text { Cutting } \\
\text { Stock } \\
\text { Problems. }\end{array}$ & $\begin{array}{l}\text { From this experiment, it was observed } \\
\text { that single-step mechanism is } \\
\text { quicker than multi-step } \\
\text { mechanisms though the distinction } \\
\text { between them was in seconds (which is } \\
\text { not acceptable for higher unit for } \\
\text { computation) to achieve optimal solution. }\end{array}$ \\
\hline 3 & $\begin{array}{l}\text { Optimal } \\
\text { Allocation } \\
\text { of Two- } \\
\text { Dimensiona } \\
1 \quad \text { Irregular } \\
\text { Shapes } \\
\text { Using an } \\
\text { Agent } \\
\text { Based } \\
\text { Approach. }\end{array}$ & $\begin{array}{l}\text { Optimization issues that are involved wit } \\
\mathrm{h} \text { finding an honest arrangement of } \\
\text { multiple objects ( } 2-\mathrm{D} \text { or } 3-\mathrm{D}) \text { during } \\
\text { a larger containing region while } \\
\text { not overlap as resolved by this method. } \\
\text { Unless and until the required criteria are } \\
\text { reached, this process continues to achieve } \\
\text { final and optimal solution. }\end{array}$ \\
\hline 4 & $\begin{array}{l}\text { Improved } \\
\text { Cut } \\
\text { Sequences } \\
\text { for Partition } \\
\text { Based } \\
\text { Placement. }\end{array}$ & $\begin{array}{l}\text { Partition based placement problem is } \\
\text { resolved and can be clearly stated that by } \\
\text { using bisections, unbalanced partitions } \\
\text { can be avoided. }\end{array}$ \\
\hline
\end{tabular}




\section{Observation from Comparative Study:}

From this above comparison table, it is observed that every approach which is taken for the solution of object cutting is dynamic in its own way and also provides precise and an optimal solution for the problem.

First paper describes the different heuristic search techniques used for allocation of two-dimensional irregular shapes.

Second paper describes two different mechanisms i.e. single step and multi-step for 2-D regular cutting stock problems.

Third paper describes the process of overlapping of multiple objects to get an optimal solution using agent based approach.

Fourth paper avoids the unbalanced partitions using different bisections to get an improved cut sequence for achieving an optimal solution.

So overall, it can be concluded that by applying or performing various techniques and approaches a precise solution can be obtained for object cutting using dynamic programming and artificial intelligence.

\section{FLOWCHART}

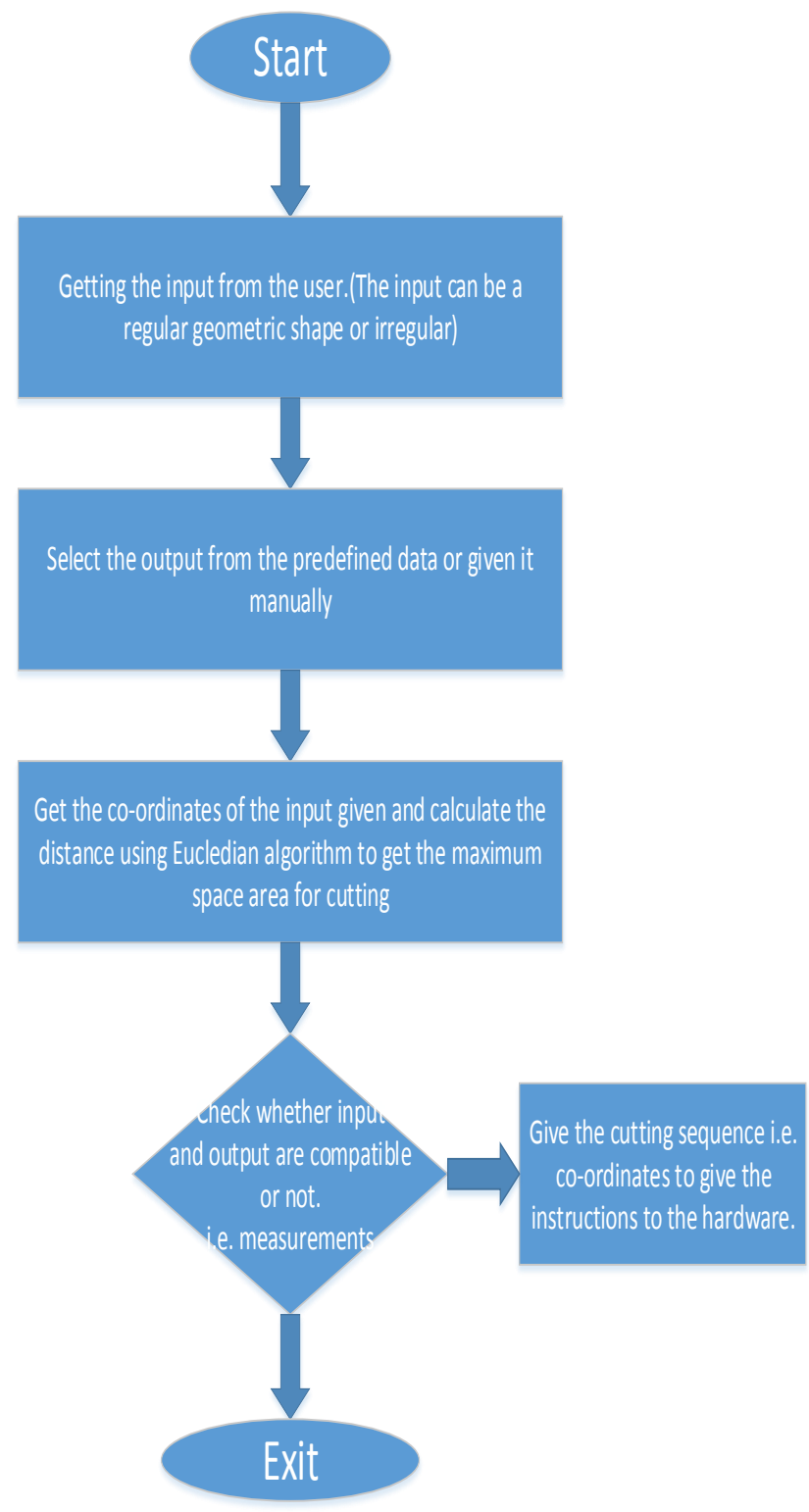

\section{CONCLUSION}

This paper covers the algorithm which is designed for object cutting problem in manufacturing industries. By comparing the survey between different research papers, the conclusion appears that by using various techniques and algorithms, the problem of stone cutting is carried out with specific and desired output. Every time different approach is applied to get optimal and precise solution for the problem. To overcome the loss of the industry, an optimal solution is required which provides the desired profit by minimizing the wastage of the materials.

\section{REFERENCES}

[1] Albano, Antonio, and Giuseppe Sapuppo. "Optimal allocation of two-dimensional irregular shapes using heuristic search methods." IEEE Transactions on Systems, Man, and Cybernetics 10.5 (1980): 242-248.

[2] Terashima-Marín, Hugo, E. J. Flores-Alvarez, and Peter Ross. "Hyper-heuristics and classifier systems for solving 2D-regular cutting stock problems." Proceedings of the 7th annual conference on Genetic and evolutionary computation. ACM, 2005.

[3] Halavati, Ramin, et al. "Optimizing Allocation of Two Dimensional Irregular Shapes using an Agent Based Approach." World Academy of Science, Engineering and Technology 11 (2005).

[4] Stoyan, Yuriy, Alexander Pankratov, and Tatiana Romanova. "Cutting and packing problems for irregular objects with continuous rotations: mathematical modelling and non-linear optimization." Journal of the Operational Research Society 67.5 (2016): 786-800.

[5] Wascher G, Hauner H and Schuma H (2007). An improved typology of cutting and packing problems. European Journal of Operational Research 183 (3) $1109-1130$

[6] Chernov N, Stoyan Y, Romanova T and Pankratov A (2012). Phi-functions for 2D objects formed by line segments and circular arcs. Advances in Operations Research doi:10.1155/2012/346358

[7] http://ieeexplore.ieee.org/document/4308483/

[8] http://link.springer.com/article/10.1007/BF01719251

[9] http://www.sciencedirect.com/science/article/pii/S08906 95502000081

[10] http://ieeexplore.ieee.org/document/4266805/

[11] http://www.sciencedirect.com/science/article/pii/037722 179090351B

[12] http://www.sciencedirect.com/science/article/pii/S00104 48500001123

[13] http://www.sciencedirect.com/science/article/pii/S09240 13605000750

[14] http://dl.acm.org/citation.cfm?id=1068115

[15] https://www.hindawi.com/journals/aor/2012/346358 